\title{
1 Spin transport in multilayer graphene away from the charge 2 neutrality point
}

4 Xin $\mathrm{He}^{\mathrm{a}}$, Yan Wen ${ }^{\mathrm{a}}$, Chenhui Zhang ${ }^{\mathrm{a}}$, Peng Li ${ }^{\mathrm{a}, \mathrm{b}}$, Dongxing Zheng ${ }^{\mathrm{a}}$, Aitian Chen ${ }^{\mathrm{a}}$, 5 Aurelien Manchon ${ }^{\mathrm{a}}$, Xixiang Zhang ${ }^{\mathrm{a}, *}$

$7 \quad{ }^{a}$ Physical Science and Engineering Division, King Abdullah University of Science and

8 Technology (KAUST), Thuwal 23955-6900, Saudi Arabia

$9{ }^{\mathrm{b}}$ Institute for Quantum Computing, Department of Electrical and Computer Engineering, 10 University of Waterloo, Waterloo N2L, Canada

13 ABSTRACT

14 Graphene is considered as a promising material in spintronics due to its long spin 15 relaxation time and long spin relaxation length. However, its spin transport properties have been 16 studied at low carrier density only, beyond which much is still unknown. In this study, we 17 explore the spin transport and spin precession properties in multilayer graphene at high carrier 18 density using ionic liquid gating. We find that the spin relaxation time is directly proportional to 19 the momentum relaxation time, indicating that the Elliott-Yafet mechanism still dominates the spin relaxation in multilayer graphene away from the charge neutrality point.

21

22 Keywords: Multilayer graphene, Ionic liquid gating, Spin transport, Elliott-Yafet mechanism 


\section{Introduction}

Due to the high charge-carrier mobility [1], weak spin-orbit coupling [2, 3], and efficiently gate-tunable transport properties [4], graphene has become a prospective material for spintronics [5-7]. When the study of spintronics in graphene just rose, the spin-relaxation time and spin-relaxation length were measured to be 170 ps and $2 \mu \mathrm{m}$ [8], respectively, which are much higher than those in the traditional materials [9], yet much lower than the theoretical predictions $[10,11]$. The poor quality of the tunneling layer [12], the polymer residues during the fabrication process [13], and the presence of scattering centers in the substrate [14, 15] are considered to be the main factors that degrade the spin transport properties of graphene. Correspondingly, several measures have been proposed to address these problems, such as the optimized insulating layer which facilitates the tunneling injection [12, 16-18], the hexagonal boron nitride $(\mathrm{hBN})$ layer which serves as a substrate or encapsulating layer to provide a smooth interface with reduced trapped charges [19], and the suspended sample structure to avoid impurity scattering $[14,20,21]$. Thanks to these efforts, the spin-relaxation time and spinrelaxation length of graphene have been improved up to $12.6 \mathrm{~ns}$ and $30.5 \mu \mathrm{m}$ [20], respectively, approaching the theoretical prediction gradually $[10,11]$. Alternatively, multilayer graphene was also proposed as a promising strategy to improve the spin transport performance since the outer layers of multilayer graphene can screen the electrical potential of impurities [22, 23]. However, this original study was limited to relatively low carrier density (close to $1.25 \times 10^{13} \mathrm{~cm}^{-2}$ ) because of the low efficiency of the traditional solid gate [23]. Hence, the conclusion that the spin relaxation is mainly due to the Elliott-Yafet mechanism was drawn only in the vicinity of the charge neutrality point [23]. When the Fermi level is shifted away from the charge neutrality point, the energy band structure and thus the electron properties will be quite different. Furthermore, the electron-electron interaction will be weakened due to the high-carrier density induced charge screening, which will influence the momentum relaxation time. Therefore, how spin transport evolves and which mechanism dominates the spin relaxation in the high-carrierdensity range of multilayer graphene is yet to be explored.

Ionic liquid (IL) gating has been widely used in 2D materials due to its high efficiency in tuning carrier density [24, 25]. It has been central to the exploration of the electronic and optical properties [26-29], for the tuning and realization of voltage-controlled superconductivity [30], 
and manipulation of the magnetic properties [31]. These studies all prove that IL gating is a mature and reliable technique.

In the present study, we explore the spin transport in multilayer graphene by tuning its carrier density using IL gating at $200 \mathrm{~K}$. In a heavily doped multilayer graphene sample, the electron and hole densities reach as high as $4.3 \times 10^{13} \mathrm{~cm}^{-2}$ and $5.7 \times 10^{13} \mathrm{~cm}^{-2}$, respectively. The spin relaxation time and the momentum relaxation time are found to follow a directly proportional relationship, which indicates that the Elliott-Yafet mechanism, rather than the D’yakonov-Perel' mechanism, plays an important role in the high-carrier density range.

62

\section{Results and analysis}

The schematic and the optical images of the device for the spin and charge transport measurements are shown in Fig. 1(a-c), while the image with IL on the device can be found in Supplementary Fig. S1. All the electrodes are made of Co/Au $(60 \mathrm{~nm} / 20 \mathrm{~nm})$. As presented in Fig. 1(a), the outer four electrodes $(1,2,5,6)$ are designed for charge transport measurement using a four-probe configuration, while the inner four electrodes $(2,3,4,5)$ are designed for spin measurements in this report were performed at $200 \mathrm{~K}$ unless otherwise specified.

75
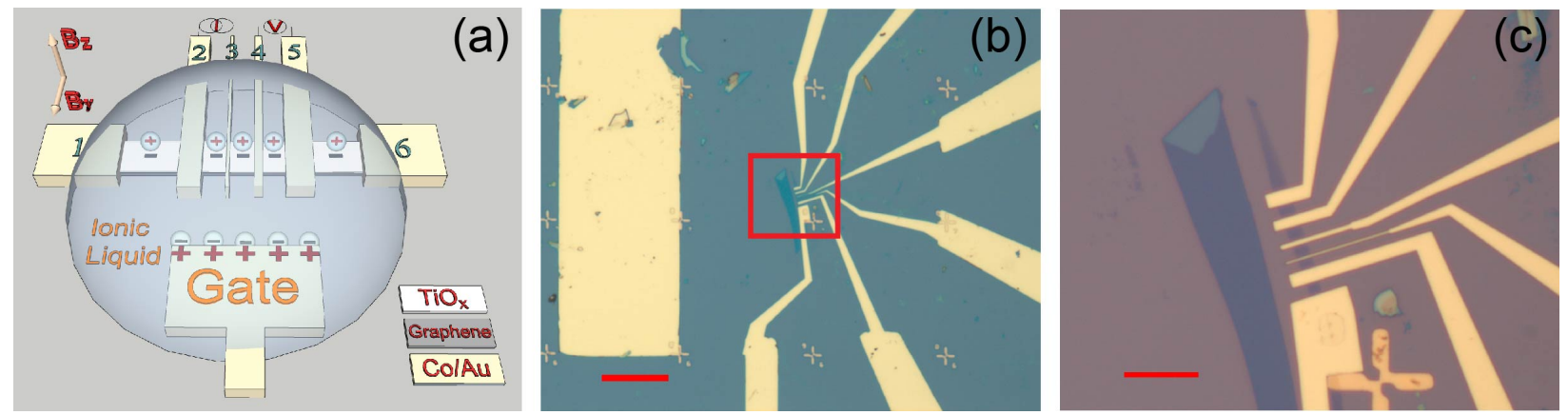
Fig. 1. (a) Schematic of a nonlocal spin-valve device under IL gating. (b) Optical image of a nonlocal spin-valve device (scale bar: $50 \mu \mathrm{m}$ ). A 12-layer graphene flake is used as the channel. (c) Zoom in on the image framed by the red open box in (b); the scale bar is $10 \mu \mathrm{m}$.

After dropping ionic liquid to cover the whole sample and setting the IL gate floating, the measurements for both spin transport and spin precession were first conducted at $300 \mathrm{~K}$. Subsequently, the system was cooled down quickly to $200 \mathrm{~K}$, the freezing point of ionic liquid [32], so as to avoid chemical reaction between graphene and ionic liquid. The same experiments were also performed at $200 \mathrm{~K}$. As shown in Fig. 1(a), under an in-plane magnetic field ( $\left.\mathrm{B}_{\mathrm{y}}\right)$, a current $\mathrm{I}$ is injected from electrode 3 (injector) to 2, and then spins accumulate underneath electrode 3 and diffuse along the graphene channel to both sides, inducing a voltage drop $\mathrm{V}_{\mathrm{NL}}$ between electrode 4 (detector) and 5 and defining the nonlocal resistance $R_{N L}=V_{N L} / I$ (spin transport) [8]. The detector probes the chemical potential of the spins impinging from the injector. Therefore, switching the magnetization configuration of the injector and detector from parallel (P) to antiparallel (AP) by sweeping the in-plane magnetic field leads to a sudden decrease of both the nonlocal voltage $\left(\Delta \mathrm{V}_{\mathrm{NL}}\right)$ and the nonlocal resistance $\left(\Delta \mathrm{R}_{\mathrm{NL}}=\Delta \mathrm{V}_{\mathrm{NL}} / \mathrm{I}\right)$, as shown in Fig. 2(a) [8, 33]. The non-local spin valve signal obtained at $200 \mathrm{~K}$ is enhanced in comparison to that measured at $300 \mathrm{~K}$ (Fig. 2(a)), due to the decreased Coulomb scattering in graphene with relatively low carrier mobility, which is consistent with previous reports [34, 35]. Applying an out-of-plane magnetic field $\left(B_{z}\right)$, a current $I$ is also injected from electrode 3 to 2 , and spins precess as they diffuse from electrode 3 to 4 , inducing a voltage drop $\mathrm{V}_{\mathrm{NL}}$ between electrode 4 and 5 and defining the nonlocal resistance $\mathrm{R}_{\mathrm{NL}}=\mathrm{V}_{\mathrm{NL}} / \mathrm{I}$ as well (spin precession). The non-local Hanle spin precession signals in both $300 \mathrm{~K}$ and $200 \mathrm{~K}$ are plotted in Supplementary Fig. S2(a) and (b), respectively. At low magnetic fields, the signal weakens because of the reduction of the spin polarization upon increasing the field from zero. When the signal corresponding to the $\mathrm{P}$ (AP) configuration decreases (increases) to zero, it indicates that the average angle of the precession reaches $90^{\circ}$ [36]. Upon increasing the field further, $\mathrm{R}_{\mathrm{NL}}$ changes sign and reaches a minimum (maximum) gradually, indicating the reversal of spins (the average precession angle reaches $180^{\circ}$ ) [36]. However, even though the vertical magnetic field was applied up to $6 \mathrm{~T}$, we could not observe the saturation of $\mathrm{R}_{\mathrm{NL}}$ that indicates the magnetization of the electrodes has completely rotated out of the plane. This is because the large-resistance 
background blurs the spin precession signal [37]. Thus, we can only estimate that $\mathrm{R}_{\mathrm{NL}}$ saturates at around 1.5 T, at which the slopes of the curves decrease suddenly (Supplementary Fig. S2).
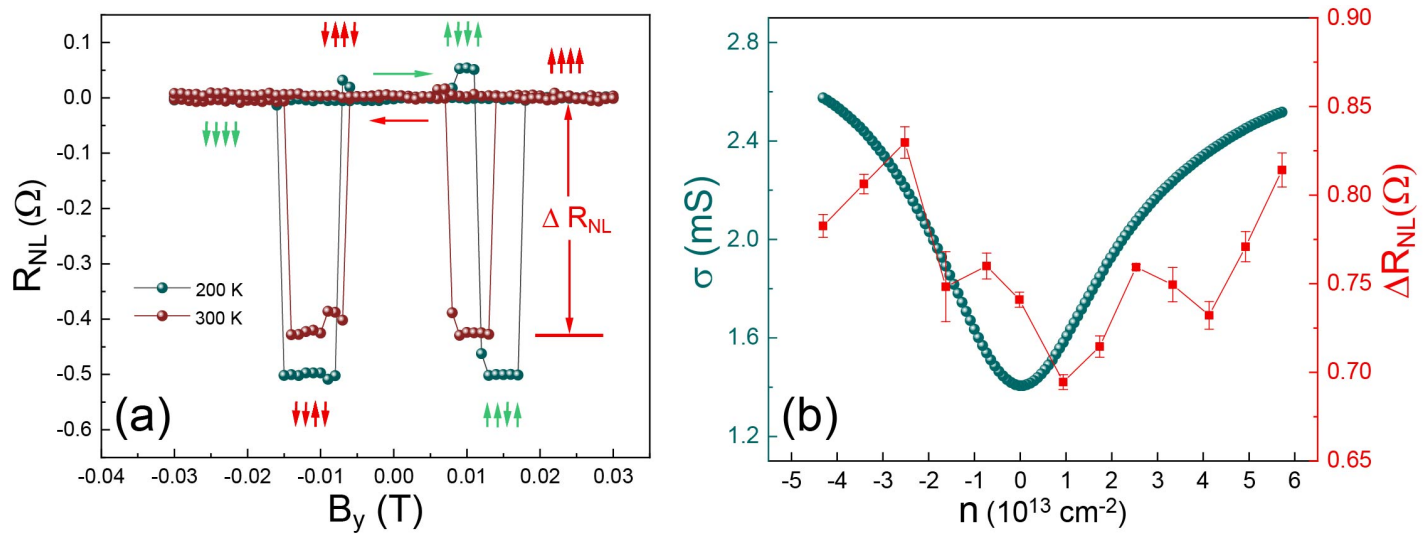

Fig. 2. (a) Non-local spin valve signal of the 12-layer graphene flack applying an in-plane magnetic field at $200 \mathrm{~K}$ and $300 \mathrm{~K}$. The magnetization configurations of the electrodes are illustrated by the arrows. The amplitude of the nonlocal resistance is defined as the change of the nonlocal resistance, $\Delta \mathrm{R}_{\mathrm{NL}}$. (b) $\sigma$ (left) and $\Delta \mathrm{R}_{\mathrm{NL}}$ (right) as functions of the carrier density $\mathrm{n}$, respectively.

To verify the validity of IL gating at $200 \mathrm{~K}$, we performed electronic measurements and then obtained the standard transfer curve of graphene, with the charge neutrality point located at $\mathrm{V}_{\mathrm{CNP}}=0.59 \mathrm{~V}$ (Fig. S3). This means that IL gating works even at $200 \mathrm{~K}$, suggesting that the molecules of IL are still mobile at $200 \mathrm{~K}$. Moreover, from Fig. S3, the mobilities ( $\mu$ ) of both electron and hole are extracted to be $242 \mathrm{~cm}^{2} / V \cdot s$ and $197 \mathrm{~cm}^{2} / V \cdot s$, respectively, by using the equation, $\mu=\Delta \sigma /\left(\mathrm{C}_{\mathrm{IL}} \Delta \mathrm{V}_{\mathrm{g}}\right)=\left(\Delta\left(\frac{\mathrm{I}_{\mathrm{ds}}}{\mathrm{V}_{\mathrm{d}}}\right) \cdot \frac{\mathrm{L}}{\mathrm{W}}\right) /\left(\mathrm{C}_{\mathrm{IL}} \Delta \mathrm{V}_{\mathrm{g}}\right)$, where $\sigma$ is the conductivity, $\mathrm{I}_{\mathrm{ds}}$ is the drain-source current, $\mathrm{V}_{\mathrm{d}}$ is the drain-source voltage, $\mathrm{V}_{\mathrm{g}}$ is the gate voltage, $\mathrm{C}_{\mathrm{IL}}$ is the capacitance per unit area of IL, L and $\mathrm{W}$ are the channel length and width, respectively [38]. $\mathrm{C}_{\mathrm{IL}}$ is obtained to be $2.86 \mu \mathrm{F} / \mathrm{cm}^{2}$ and $2.55 \mu \mathrm{F} / \mathrm{cm}^{2}$ for the electron and hole, respectively (Supplementary Note 1), much lower than the values reported previously [26, 39]. There are two factors leading to the small $\mathrm{C}_{\mathrm{IL}}$ : the extra $\mathrm{TiO}_{\mathrm{X}}$ layer and the screening effect in graphene. First, the inserted $\mathrm{TiO}_{\mathrm{X}}$ layer (about $1 \mathrm{~nm}$ thick) increases the effective dielectric thickness of the geometrical capacitance $\mathrm{C}_{\mathrm{g}}$, which is defined by $\mathrm{C}_{\mathrm{g}}=\varepsilon_{\mathrm{IL}} \varepsilon_{0} / \mathrm{d}$, where $\varepsilon_{\mathrm{IL}}$ is the relative permittivity of ionic liquid, $\varepsilon_{0}$ is the vacuum permittivity, and $d$ is the dielectric thickness [40]. Second, the charges distributed in the first 3 or 4 layers screen the electric field and quench its penetration, and then 
$132 \mathrm{C}_{\mathrm{g}}$ saturates to a constant as the layer number is larger than 4 [40]. In Fig. 2(b), the IL gate 133 voltage is transformed to the carrier concentration $\mathrm{N}$ by using the formula, $\mathrm{N}=\mathrm{C}_{\mathrm{IL}} \frac{\left(\mathrm{V}_{\mathrm{g}}-\mathrm{V}_{\mathrm{CNP}}\right)}{\mathrm{e}}$, 134 where $\mathrm{e}<0$ is the elementary charge $[32,41]$. Then the highest electron (hole) density can be 135 calculated to be $4.3 \times 10^{13} \mathrm{~cm}^{-2}\left(5.7 \times 10^{13} \mathrm{~cm}^{-2}\right)$. Also in Fig. 2(b), the changes of the non-local resistances were extracted by performing the non-local spin transport at a series of carrier 137 densities, as a comparison to the carrier density dependence of the conductivity. The relationship 138 between $\Delta \mathrm{R}_{\mathrm{NL}}$ and $\sigma$ in Fig. 2(b) follows neither $\Delta R_{N L} \sim \sigma$ nor $\Delta R_{N L} \sim \frac{1}{\sigma}$, implying that the 139 electrode contact could be a pinhole type [12]. As discussed in the previous study, the contact 140 interface mainly affects the polarization of the injected current [23], and the pinhole-type 141 contacts could also create long spin relaxation time and spin relaxation length [20], thus the 142 contacts barely influence the spin diffusion and spin relaxation in our study.
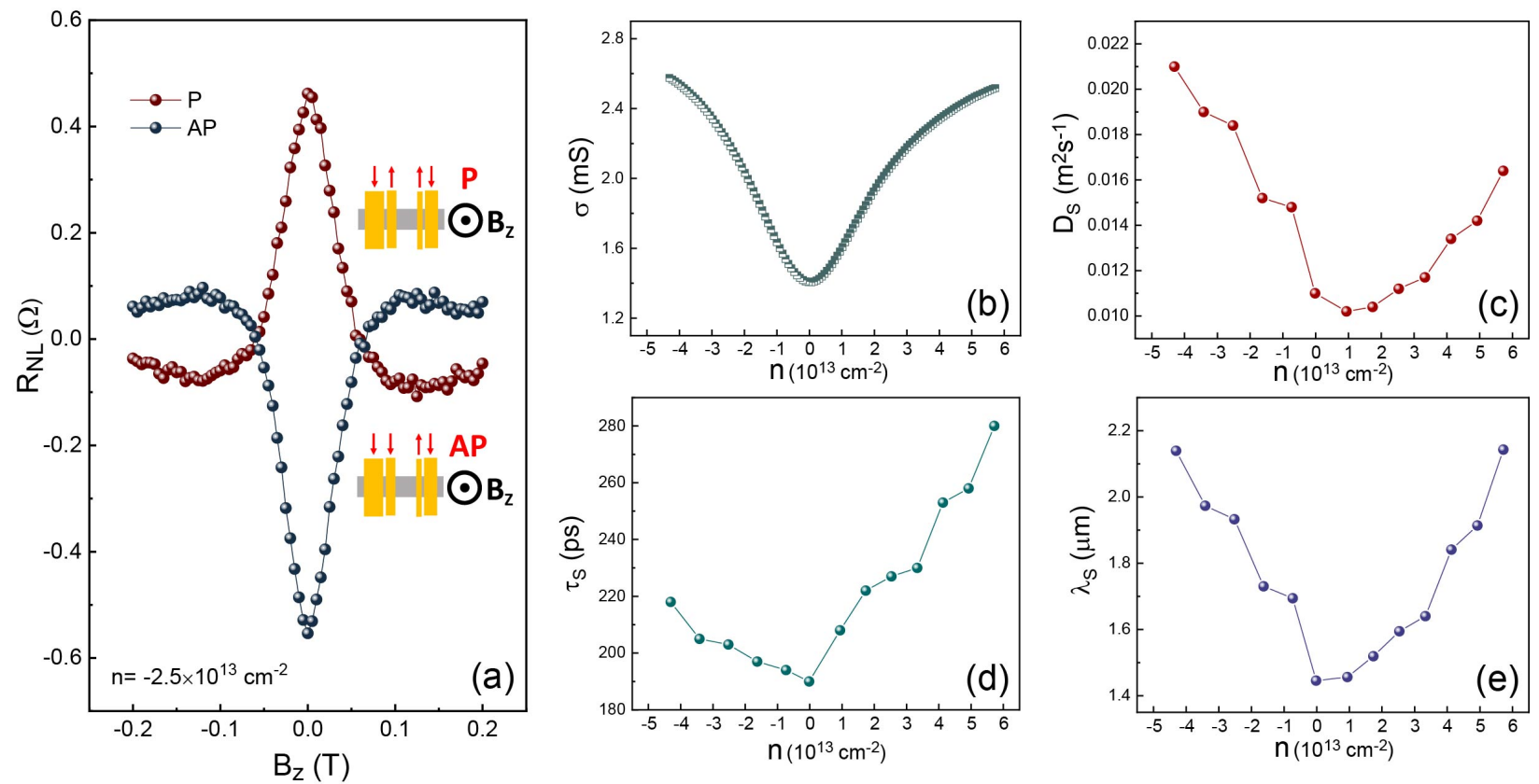

145

146

147

148

149

150

Fig. 3. (a) Nonlocal Hanle spin precession signal of the 12-layer graphene flake at the electron density of $2.5 \times 10^{13} \mathrm{~cm}^{-2}, \mathrm{P}$ and AP magnetizations of the central two electrodes are illustrated in the insets. $\sigma(\mathrm{b}), \mathrm{D}_{\mathrm{S}}(\mathrm{c}), \tau_{\mathrm{S}}(\mathrm{d})$ and $\lambda_{\mathrm{S}}(\mathrm{e})$ as a function of the carrier density $\mathrm{n}$, respectively.

The spin precession at different carrier densities is studied applying a perpendicular magnetic field at $200 \mathrm{~K}$, as shown in Fig. 3. As an example, Fig. 3(a) shows the nonlocal Hanle 
151 spin precession signals with both $\mathrm{P}$ and $\mathrm{AP}$ magnetization of the central electrodes at $\mathrm{V}_{\mathrm{g}}=2 \mathrm{~V}$, 152 corresponding to the electron density of $2.5 \times 10^{13} \mathrm{~cm}^{-2}$. Since the magnetic field is applied 153 perpendicular to the plane, the spins with in-plane orientation begin to precess, following the 154 Bloch equation for the spin accumulation $\mu_{S}, D_{S} \nabla^{2} \mu_{S}-\frac{\mu_{S}}{\tau_{S}}+\omega_{L} \times \mu_{S}=0$ [23]. The first, second 155 and third term describes the spin diffusion, spin relaxation, and spin precession, respectively, with $D_{S}$ is the spin diffusion coefficient, $\tau_{S}$ is the spin relaxation time, and $\omega_{L}=\frac{g \mu_{B} H}{\hbar}$ is the 157 Larmor frequency ( $\mathrm{g}=2$ is the effective Lande factor, $\mu_{\mathrm{B}}$ is the Bohr magneton, and $\mathbf{H}$ is the out158 of-plane magnetic field). Moreover, $\mathrm{D}_{\mathrm{S}}$ and $\tau_{\mathrm{S}}$ can be extracted by fitting the Hanle precession 159 signal at each carrier density with the solution of the above Bloch 160 equation, $\mathrm{R}_{\mathrm{NL}}^{\mathrm{P}(\mathrm{AP})}=( \pm) \mathrm{S}_{0} \int \frac{1}{\sqrt{4 \pi \mathrm{D}_{\mathrm{S}} \mathrm{t}}} \exp \left(-\frac{\mathrm{L}^{2}}{4 \mathrm{D}_{\mathrm{S}}}\right) \cos \left(\omega_{\mathrm{L}} \mathrm{t}\right) \exp \left(-\frac{\mathrm{t}}{\tau_{\mathrm{S}}}\right) \mathrm{dt}$ [42] (where $R_{N L}^{P(A P)}$ is the 161 nonlocal magnetoresistance, $+(-)$ sign is for the $\mathrm{P}(\mathrm{AP})$ state, $\mathrm{S}_{0}$ is the amplitude, and $\mathrm{L}$ is the 162 channel length between the central two electrodes), while the spin diffusion length $\lambda_{\mathrm{S}}$ can be 163 calculated by $\lambda_{S}=\sqrt{D_{S} \tau_{S}}$ [23]. Note that before fitting the result, the $\mathrm{P}$ curve of the Hanle 164 effect was subtracted from the AP curve (e.g., Fig. 3(a)) to remove the background that is not 165 related to the spin signal [23]. Therefore, the relationships between $\sigma$ and $n, D_{S}$ and $n, \tau_{S}$ and $n$, 166 and $\lambda_{\mathrm{S}}$ and $\mathrm{n}$ are presented in Fig. 3(b-e), respectively. $\mathrm{D}_{\mathrm{S}}, \tau_{\mathrm{S}}$, and $\lambda_{\mathrm{S}}$ all diminish with 167 decreasing the electron or the hole density, and reach the minimum values in the vicinity of the 168 charge neutrality point, resembling the relationship between $\sigma$ and $n$. Compared with the 169 previous data obtained using the traditional solid gate in multilayer graphene [23], these 170 parameters show more significant changes because of the stronger carrier density modulation 171 induced by the IL gating. The asymmetry of $\mathrm{D}_{\mathrm{S}}$ for electrons and holes in Fig. 3(c) may be due to 172 the nonuniformities in the carrier density [43], while the asymmetry of $\tau_{\mathrm{S}}$ in Fig. 3(d) could be 173 traced to the different impacts of the spin scatterings to the electrons and holes. 

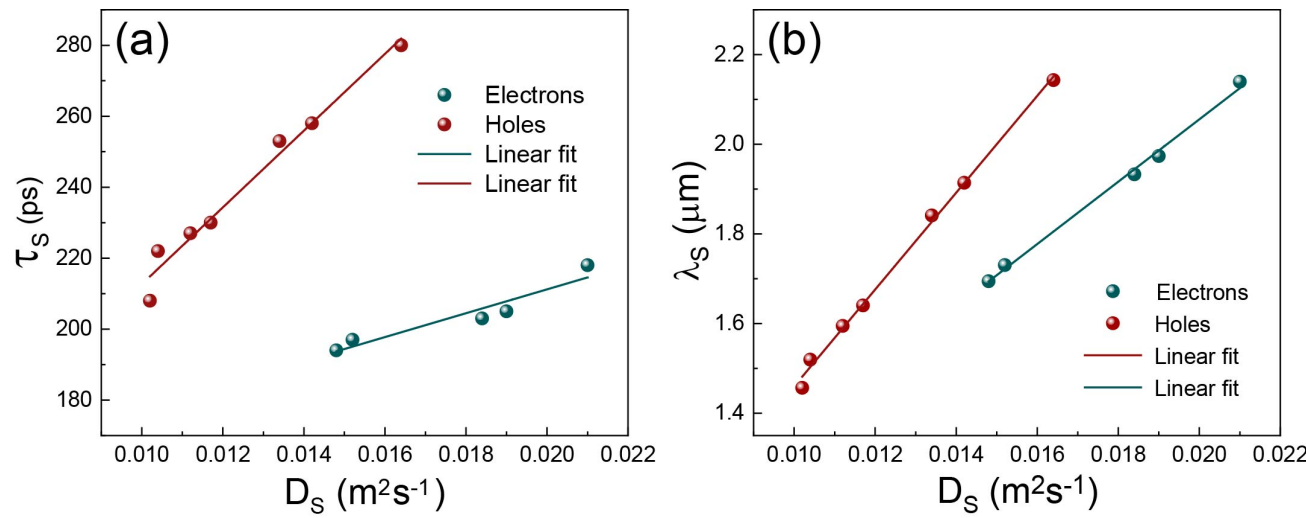

Fig. 4. (a) Linear relationship between $\tau_{S}$ and $D_{S}$, extracted from Fig. 3(c) and (d). (b) Linear relationship between $\lambda_{S}$ and $D_{S}$, extracted from Fig. 3(c) and (e).

In semiconductors, there are two main mechanisms that contribute to spin relaxation. The first one is the Elliott-Yafet mechanism, which stems from momentum transfer between spin and orbital angular momenta during momentum scattering events, resulting in $\tau_{\mathrm{S}}$ directly proportional to $\tau_{\mathrm{P}}\left(\tau_{\mathrm{P}}\right.$ is the momentum relaxation time) $[43,44]$. The second one is the D'yakonov-Perel' mechanism, which stems from scattering-induced destructive interference of spins coherently precessing around the spin-orbit field in the band structure. In this case, $\tau_{\mathrm{S}}$ is inversely proportional to $\tau_{\mathrm{P}}[43,44]$. Thus, to identify which spin scattering mechanism dominates the spin relaxation, we need to determine the relationship between $\tau_{\mathrm{S}}$ and $\tau_{\mathrm{P}}$. Fig. 4(a) shows the linear dependence between $\tau_{S}$ and $D_{S}\left(\tau_{S} \propto D_{S}\right)$, extracted from Fig. 3(c) and (d); while in Fig. 4(b), $\lambda_{S}$ and $\mathrm{D}_{\mathrm{S}}$ are also in direct proportion, extracted from Fig. 3(c) and (e). The larger slopes for the hole regimes indicates the averagely lower spin-flip probability for holes in each scattering [23]. These relationships are consistent with the previous studies in both monolayer and multilayer graphene [23, 43, 45]. Combing the facts that $D_{S} \propto D_{P}\left(D_{P}\right.$ is the momentum diffusion coefficient) [23] and $D_{P} \propto \tau_{P}[42,43]$, we obtain $\tau_{S} \propto \tau_{P}$. This indicates that the Elliott-Yafet mechanism still plays a more important role in the spin relaxation process of multilayer graphene at high carrier density [43, 44]. Moreover, a large perpendicular electric field can lead to a considerable in-plane Rashba spin-orbit field, which will affect the spin transport properties, i.g., the spin relaxation time [37]. In our experiment, the gate voltage up to $3 \mathrm{~V}$ is applied, and the electric field penetrates to the first 3 or 4 layers of graphene. Considering the ionic liquid molecule (diameter $1 \mathrm{~nm}$ ) and the $\mathrm{TiO}_{\mathrm{X}}$ layer (thickness $\sim 1 \mathrm{~nm}$ ), the electric field reaches around 
$1 \mathrm{~V} / \mathrm{nm}$ in graphene theoretically. However, we do not see the deviation of the spin relaxation time in Fig. 3(d), which means that the induced spin-orbit field is quite weak, because of the strong screening of the charges to the applied electric field.

\section{Outlook}

Recently, to realize more functions in graphene-based spin devices, the heterostructures such as graphene/topological insulators [46], graphene/magnetic insulator [47], and graphene/Transition metal dichalcogenides [48] have been constructed. These heterostructures combine both the excellent spin properties of graphene and the exotic properties of other materials via proximity effect. Moreover, the nano-graphene based spin device is also drawing more attention [49-52]. Due to the weak tuning capability of the traditional solid gate, the gate voltage that is used to switch on/off the spin current is always as high as several tens of volts. We believe that the IL gate can be used to improve the gating effect in these devices and allow them to work practically at low voltages.

\section{Conclusion}

In conclusion, we provide more insights into the spin dynamics in multilayer graphene and verified that IL gating is a powerful technique investigating spintronics in 2D materials. After applying IL gate, the electron and hole densities in multilayer graphene are increased up to $4.3 \times 10^{13} \mathrm{~cm}^{-2}$ and $5.7 \times 10^{13} \mathrm{~cm}^{-2}$, respectively. The spin relaxation time and the momentum relaxation time are found to follow a directly proportional relationship, indicating that the ElliottYafet mechanism still dominates the spin relaxation in multilayer graphene at high carrier density.

\section{Methods}

Multilayer graphene samples were exfoliated from natural graphite onto $\mathrm{SiO}_{2} / \mathrm{Si}(300 \mathrm{~nm}$ / $500 \mu \mathrm{m}$ ) substrate. To avoid the extra etching process, the samples which were relatively long and narrow were located by optical microscope (Carl Zeiss Imager.A2 Vario with AxioCam 
$226 \mathrm{HRc}$ ), and then their thicknesses were confirmed by atomic force microscope (Asylum Research

227 MFP-3D) using tapping mode. After that, $0.8 \mathrm{~nm}$ Ti was evaporated onto the samples using e228 beam evaporator, and then they were exposed in air for $0.5 \mathrm{~h}$ to form an oxidation layer (the $229 \mathrm{TiO}_{\mathrm{X}}$ layer not only plays a role of tunneling layer to conquer the conductance mismatch 230 problem but also prevents the sample from reacting with ionic liquid) [53]. Subsequently, six 231 electrodes were fabricated for each sample using e-beam lithography (EBL) and followed e232 beam evaporation of $\mathrm{Co} / \mathrm{Au}(60 \mathrm{~nm} / 20 \mathrm{~nm})$. After the lift-off process, the samples were

233 immersed in chloroform $(60,4$ hours $)$ to remove the polymer residues to facilitate the usage of 234 IL, which was (N,N-diethyl-N-methyl-N-(2-methoxyethyl)ammonium 235 Bis(trifluoromethanesulfonyl)imide (DEME-TFSI)) (Kanto Chemical Co., Inc.). With Keithley $2362636 \mathrm{~B}$ applying IL-gate voltage, an alternating current of $20 \mu \mathrm{A}$ at $13 \mathrm{HZ}$ was applied by 237 Keithley 6221 and the voltage was measured by lock-in amplifier SR830 in the spin transport 238 measurements, while the alternating current was changed to $1 \mu \mathrm{A}$ in the electronic transport 239 measurements. The transfer curves in the supplementary material were measured using the 240 Keithley 4200-SCS semiconductor characterization system.

\section{Conflicts of interest}

243 The authors declare no competing financial interests.

\section{Acknowledgments}

246 This publication is based on research supported by the King Abdullah University of Science and 247 Technology (KAUST), under Award Nos. OSR-2018-CRG7-3717 and OSR-2017-CRG6-3427.

\section{Appendix A. Supplementary data}

250 Supplementary data to this article can be found online at XXXXXXXXX. 


\section{References}

252

253

254

255

256

257

258

259

260

261

262

263

264

265

266

267

268

269

270

271

272

273

274

275

276

277

278

279

280

281

282

283

284

285

286

287

288

289

290

291

292

293

294

295

296

[1] C.R. Dean, A.F. Young, I. Meric, C. Lee, L. Wang, S. Sorgenfrei, et al., Boron nitride substrates for highquality graphene electronics, Nat. Nanotechnol. 5 (2010) 722-726.

[2] M. Gmitra, S. Konschuh, C. Ertler, C. Ambrosch-Draxl, J. Fabian, Band-structure topologies of graphene: Spin-orbit coupling effects from first principles, Phys. Rev. B 80 (2009) 235431.

[3] H. Min, J.E. Hill, N.A. Sinitsyn, B.R. Sahu, L. Kleinman, A.H. MacDonald, Intrinsic and Rashba spin-orbit interactions in graphene sheets, Phys. Rev. B 74 (2006) 165310.

[4] F. Schwierz, Graphene transistors, Nat. Nanotechnol. 5 (2010) 487-496.

[5] M.V. Kamalakar, C. Groenveld, A. Dankert, S.P. Dash, Long distance spin communication in chemical vapour deposited graphene, Nat. Commun. 6 (2015) 6766.

[6] S. Roche, Aring, J. kerman, B. Beschoten, J.C. Charlier, M. Chshiev, et al., Graphene spintronics: the European Flagship perspective, 2d Materials 2 (2015) 030202.

[7] D. Khokhriakov, B. Karpiak, A.M. Hoque, S.P. Dash, Two-dimensional spintronic circuit architectures on large scale graphene, Carbon 161 (2020) 892-899.

[8] N. Tombros, C. Jozsa, M. Popinciuc, H.T. Jonkman, B.J. van Wees, Electronic spin transport and spin precession in single graphene layers at room temperature, Nature 448 (2007) 571-574.

[9] J. Sinova, S.O. Valenzuela, J. Wunderlich, C.H. Back, T. Jungwirth, Spin Hall effects, Rev. Mod. Phys. 87 (2015) 1213-1259.

[10] D. Huertas-Hernando, F. Guinea, A. Brataas, Spin-orbit-mediated spin relaxation in graphene, Phys. Rev. Lett. 103 (2009) 146801.

[11] D. Pesin, A.H. MacDonald, Spintronics and pseudospintronics in graphene and topological insulators, Nat. Mater. 11 (2012) 409-416.

[12] W. Han, K. Pi, K.M. McCreary, Y. Li, J.J.I. Wong, A.G. Swartz, et al., Tunneling spin injection into single layer graphene, Phys. Rev. Lett. 105 (2010) 167202.

[13] A. Avsar, I.J. Vera-Marun, J.Y. Tan, G.K.W. Koon, K. Watanabe, T. Taniguchi, et al., Electronic spin transport in dual-gated bilayer graphene, NPG Asia Mater. 8 (2016) e274.

[14] M.H.D. Guimaraes, A. Veligura, P.J. Zomer, T. Maassen, I.J. Vera-Marun, N. Tombros, et al., Spin transport in high-quality suspended graphene devices, Nano Lett. 12 (2012) 3512-3517.

[15] P.J. Zomer, M.H.D. Guimaraes, N. Tombros, B.J. van Wees, Long-distance spin transport in highmobility graphene on hexagonal boron nitride, Phys. Rev. B 86 (2012) 161416(R).

[16] M.V. Kamalakar, A. Dankert, J. Bergsten, T. Ive, S.P. Dash, Enhanced tunnel spin injection into graphene using chemical vapor deposited hexagonal boron nitride, Sci Rep 4 (2014) 6146.

[17] S. Singh, J. Katoch, J.S. Xu, C. Tan, T.C. Zhu, W. Amamou, et al., Nanosecond spin relaxation times in single layer graphene spin valves with hexagonal boron nitride tunnel barriers, Appl. Phys. Lett. 109 (2016) 122411.

[18] M.V. Kamalakar, A. Dankert, P.J. Kelly, S.P. Dash, Inversion of Spin Signal and Spin Filtering in Ferromagnet vertical bar Hexagonal Boron Nitride-Graphene van der Waals Heterostructures, Sci Rep 6 (2016) 21168.

[19] M. Gurram, S. Omar, B.J. van Wees, Electrical spin injection, transport, and detection in graphenehexagonal boron nitride van der Waals heterostructures: progress and perspectives, 2D Materials 5 (2018) 032004.

[20] M. Drogeler, C. Franzen, F. Volmer, T. Pohlmann, L. Banszerus, M. Wolter, et al., Spin lifetimes exceeding $12 \mathrm{~ns}$ in graphene nonlocal spin valve devices, Nano Lett. 16 (2016) 3533-3539.

[21] M. Drogeler, F. Volmer, M. Wolter, B. Terres, K. Watanabe, T. Taniguchi, et al., Nanosecond spin lifetimes in single- and few-layer graphene-hBN heterostructures at room temperature, Nano Lett. 14 (2014) 6050-6055. 
[22] L. Li, I. Lee, D. Lim, S. Rathi, M. Kang, T. Uemura, et al., Spin diffusion and non-local spin-valve effect in an exfoliated multilayer graphene with a Co electrode, Nanotechnology 27 (2016) 335201.

[23] T. Maassen, F.K. Dejene, M.H.D. Guimaraes, C. Jozsa, B.J. van Wees, Comparison between charge and spin transport in few-layer graphene, Phys. Rev. B 83 (2011) 115410.

[24] H.W. Du, X. Lin, Z.M. Xu, D.W. Chu, Electric double-layer transistors: a review of recent progress, J. Mater. Sci. 50 (2015) 5641-5673.

[25] T. Fujimoto, K. Awaga, Electric-double-layer field-effect transistors with ionic liquids, Phys. Chem. Chem. Phys. 15 (2013) 8983-9006.

[26] J.T. Ye, M.F. Craciun, M. Koshino, S. Russo, S. Inoue, H.T. Yuan, et al., Accessing the transport properties of graphene and its multilayers at high carrier density, Proc. Natl. Acad. Sci. U. S. A. 108 (2011) $307 \quad 13002-13006$. with ambipolar ionic liquid-gated transistors, Nano Lett. 12 (2012) 5218-5223.

[28] X. He, N. Tang, L. Gao, J.X. Duan, Y.W. Zhang, F.C. Lu, et al., Formation of p-n-p junction with ionic liquid gate in graphene, Appl. Phys. Lett. 104 (2014) 143102.

[29] X. He, Z.H. Zhang, C.H. Zhang, Y. Yang, M. Hu, W.K. Ge, et al., Exploration of exciton behavior in atomically thin WS2 layers by ionic gating, Appl. Phys. Lett. 113 (2018) 013104.

[30] Y. Saito, T. Nojima, Y. Iwasa, Gate-induced superconductivity in two-dimensional atomic crystals, Supercond. Sci. Technol. 29 (2016) 093001.

[31] Y.J. Deng, Y.J. Yu, Y.C. Song, J.Z. Zhang, N.Z. Wang, Z.Y. Sun, et al., Gate-tunable room-temperature ferromagnetism in two-dimensional Fe3GeTe2, Nature 563 (2018) 94-99.

[32] M.M. Perera, M.W. Lin, H.J. Chuang, B.P. Chamlagain, C.Y. Wang, X.B. Tan, et al., Improved carrier mobility in few-layer MoS2 field-effect transistors with ionic-liquid gating, ACS Nano 7 (2013) 4449-4458. [33] F.J. Jedema, A.T. Filip, B.J. van Wees, Electrical spin injection and accumulation at room temperature in an all-metal mesoscopic spin valve, Nature 410 (2001) 345-348.

[34] T. Maassen, J.J. van den Berg, N. Ijbema, F. Fromm, T. Seyller, R. Yakimova, et al., Long spin relaxation times in wafer scale epitaxial graphene on SiC(0001), Nano Lett. 12 (2012) 1498-1502.

[35] T. Yamaguchi, R. Moriya, S. Masubuchi, K. Iguchi, T. Machida, Spin relaxation in weak localization regime in multilayer graphene spin valves, Jpn. J. Appl. Phys. 52 (2013) 040205.

[36] F.J. Jedema, H.B. Heersche, A.T. Filip, J.J.A. Baselmans, B.J. van Wees, Electrical detection of spin precession in a metallic mesoscopic spin valve, Nature 416 (2002) 713-716.

[37] M.H.D. Guimaraes, P.J. Zomer, J. Ingla-Aynes, J.C. Brant, N. Tombros, B.J. van Wees, Controlling spin relaxation in hexagonal $\mathrm{BN}$-encapsulated graphene with a transverse electric field, Phys. Rev. Lett. 113 (2014) 086602.

[38] T. Kim, H. Kirn, S.W. Kwon, Y. Kim, W.K. Park, D.H. Yoon, et al., Large-scale graphene micropatterns via self-assembly-mediated process for flexible device application, Nano Lett. 12 (2012) 743-748.

[39] Y.J. Zhang, J.T. Ye, Y. Matsuhashi, Y. Iwasa, Ambipolar MoS2 thin flake transistors, Nano Lett. 12 (2012) 1136-1140.

[40] E. Uesugi, H. Goto, R. Eguchi, A. Fujiwara, Y. Kubozono, Electric double-layer capacitance between an ionic liquid and few-layer graphene, Sci Rep 3 (2013) 1595.

[41] R. Misra, M. McCarthy, A.F. Hebard, Electric field gating with ionic liquids, Appl. Phys. Lett. 90 (2007) 052905.

[42] K. Pi, W. Han, K.M. McCreary, A.G. Swartz, Y. Li, R.K. Kawakami, Manipulation of spin transport in graphene by surface chemical doping, Phys. Rev. Lett. 104 (2010) 187201.

[43] C. Jozsa, T. Maassen, M. Popinciuc, P.J. Zomer, A. Veligura, H.T. Jonkman, et al., Linear scaling between momentum and spin scattering in graphene, Phys. Rev. B 80 (2009) 241403(R).

[44] A. Avsar, T.Y. Yang, S. Bae, J. Balakrishnan, F. Volmer, M. Jaiswal, et al., Toward wafer scale fabrication of graphene based spin valve devices, Nano Lett. 11 (2011) 2363-2368. 
[45] W. Han, R.K. Kawakami, Spin relaxation in single-layer and bilayer graphene, Phys. Rev. Lett. 107 346 (2011) 047207.

347 [46] D. Khokhriakov, A.W. Cummings, K. Song, M. Vila, B. Karpiak, A. Dankert, et al., Tailoring emergent 348 spin phenomena in Dirac material heterostructures, Science Advances 4 (2018) eaat9349.

349 [47] B. Karpiak, A.W. Cummings, K. Zollner, M. Vila, D. Khokhriakov, A.M. Hoque, et al., Magnetic proximity in a van der Waals heterostructure of magnetic insulator and graphene, 2d Materials 7 (2020) 351015026.

352 [48] A. Dankert, S.P. Dash, Electrical gate control of spin current in van der Waals heterostructures at 353 room temperature, Nat. Commun. 8 (2017) 16093.

354 [49] Z.Q. Fan, F. Xie, X.W. Jiang, Z.M. Wei, S.S. Li, Giant decreasing of spin current in a single molecular 355 junction with twisted zigzag graphene nanoribbon electrodes, Carbon 110 (2016) 200-206.

356 [50] Z.Q. Fan, W.Y. Sun, X.W. Jiang, Z.H. Zhang, X.Q. Deng, G.P. Tang, et al., Redox control of magnetic 357 transport properties of a single anthraquinone molecule with different contacted geometries, Carbon $358113(2017) 18-25$.

359 [51] Z.Q. Fan, W.Y. Sun, Z.H. Zhang, X.Q. Deng, G.P. Tang, H.Q. Xie, Symmetry-dependent spin transport 360 properties of a single phenalenyl or pyrene molecular device, Carbon 122 (2017) 687-693.

[52] T. Enoki, M. Kiguchi, Magnetism of Nanographene-Based Microporous Carbon and Its Applications: Interplay of Edge Geometry and Chemistry Details in the Edge State, Physical Review Applied 9 (2018) 037001.

364 [53] C. Song, J.H. Miao, F. Li, Y.N. Yan, B. Cui, F. Pan, Anomalous Hall effect in one monolayer cobalt with 365 electrical manipulation, J. Alloy. Compd. 696 (2017) 315-320. 